\title{
MULTIPLE FIBERS ON RATIONAL ELLIPTIC SURFACES
}

\author{
BRIAN HARBOURNE AND WILLIAM E. LANG
}

\begin{abstract}
Our main result, Theorem (0.1), classifies multiple fibers on rational elliptic surfaces over algebraically closed fields of arbitrary characteristic. One result of this is the existence in positive characteristics of tame multiple fibers of additive type for several of the Kodaira fiber-types for which no examples were previously known.
\end{abstract}

0. Introduction. We work in this paper over an algebraically closed field $k$ of arbitrary characteristic $p$. An elliptic surface $f: X \rightarrow B$ is a fibration of a complete smooth surface $X$ over a complete smooth curve $B$ such that the generic fiber is a smooth curve of genus one. It is natural to wonder what kind of special fibers arise. We answer this in the case of rational surfaces, giving as a result examples of tame multiple fibers of additive type for every $p>0$ and for all such types except $I_{b}^{*}, b>4$ (cf. §5). To our knowledge, the only example heretofore recognized is of multiplicity 2 and Kodaira type $I_{0}^{*}$, due to Katsura [Ka, L1] (although since our results were obtained we have seen a preprint of Katsura and Ueno [KU] giving other examples, mostly for $p \neq 2,3$ and Kodaira type not $\left.I_{b}^{*}, b>0\right)$.

Any special fiber is an effective divisor on $X$ and so can be written as $m F=$ $m \sum n_{j} C_{j}$, where each curve $C_{j}$ is reduced and irreducible and the coefficients are positive integers with g.c.d. $\left(\left\{n_{j}\right\}\right)=1$. The positive integer $m$ is the multiplicity of the fiber $m F$ which is said to be a multiple fiber whenever $m>1$.

The form of the divisor $F=\sum n_{j} C_{j}$ is very restricted. Such a divisor is an indecomposable curve of canonical type [Mu]; a topological classification of all such curves has been given by Kodaira [Ko] (cf. §5). We refer to the classes which arise in this classification as the Kodaira (fiber-)types. More generally, the Kodaira type of the fiber $m F$ is $m T$, where $T$ is the type of $F$.

Over an algebraically closed field $k$ it is known that any multiple fiber $m F$ of multiplicity $m$ on a surface $X$ is of the form $m T$, where $T$ is a Kodaira type and $m$ is divisible by (and in characteristic 0 equal to) the order of the normal bundle $O_{X}(F) \otimes O_{F} \in \mathrm{Pic}^{0} F$ of $F$ in $X$, but the extent to which these conditions are sufficient for there to occur on some elliptic surface a multiple fiber of type $m T$ is not known. Of particular interest to us here are the tame multiple fibers of additive type, these being fibers $m F$ of multiplicity $m$ where $\mathrm{Pic}^{0} F$ is the additive group $\mathbf{G}_{a}$ of the ground field $k$ and $m$ equals the order in $\mathbf{G}_{a}$ of $O_{X}(F) \otimes O_{F}$. These are possible only if $p$, the characteristic of $k$, is positive (since $\mathbf{G}_{a}$ only then has $p$-torsion), but their occurrence is poorly understood.

Received by the editors March 21, 1987.

1980 Mathematics Subject Classification (1985 Revision). Primary 14J25, 14M20, 14D99.

Key words and phrases. Rational, elliptic, surface, multiple fiber, positive characteristic.

Both authors were partially supported by NSF grants. 
In contrast to this situation, over the complex field $k=\mathbb{C}$ it is well understood what multiplicities occur. Fibers of additive type, which are precisely the simply connected ones, can occur only with multiplicity one. For a nonsimply connected fiber type $T, \mathrm{Pic}^{0} T$ has torsion of all orders and Kodaira shows via his logarithmic transform construction that every positive integer occurs as a multiplicity.

Recently W. E. Lang [L4] has constructed an analogue in positive characteristics of the logarithmic transform by means of which a surface with specified multiple fibers can be constructed if each multiple fiber occurs on a rational surface. This motivates the principal result, Theorem (0.1), of the present paper, which gives a complete determination of the types $m T$ which appear as fibers on rational minimal elliptic surfaces. Since multiple fibers on a rational surface are tame (cf. (3.1)), this brings to light many more examples of tame multiple fibers of additive type:

THEOREM (0.1). There is a rational minimal elliptic surface on which a fiber of type $m T, m>0$, appears if and only if $T$ has no more than nine irreducible components and at the same time one of the following conditions holds:

(a) $T$ is the type of a smooth fiber; or

(b) $T$ is of additive type and $m$ is either 1 or $\operatorname{char}(k)$; or

(c) $T$ is neither the type of a smooth fiber nor of additive type (i.e., $T$ is of multiplicative type (cf. §5)) and $m$ is any positive integer not a multiple of $\operatorname{char}(k)$.

The major ingredient used in the proof is the construction and analysis of a birational transformation which we call a Halphen transformation, the effect of which is to take a fiber of a given pencil and to create a new pencil in which a multiple of that fiber occurs as a fiber.

The paper divides naturally into four parts. The first, comprising $\S \S 1$ through 6 , recall standard facts we need later. The second, comprising $\S 7$, defines and studies the properties of the Halphen transformation, from which a proof of Theorem (0.1) follows easily for any characteristic but 2 or 3 ; the possibility of quasi-elliptic fibrations in characteristic 2 or 3 complicates the proof. The third, comprising $\S 8$, adduces facts about quasi-elliptic fibrations. The fourth, comprising $\S 9$, gives a proof of Theorem (0.1) in all characteristics, providing in the process some explicit examples of elliptic fibrations with multiple fibers of additive type. Moreover, our proof is completely constructive.

1. Numerically elliptic surfaces. Because of the occurrence of quasi-elliptic surfaces in characteristics 2 and 3 , it is convenient for a unified treatment to define a numerically elliptic surface to be a fibration $f: X \rightarrow B$ of a smooth surface $X$ over a smooth curve $B$ such that $f_{*} O_{X}$ is isomorphic to $O_{B}$ and such that the generic fiber is a reduced irreducible curve of arithmetic genus 1 . It is said to be elliptic if this generic fiber is smooth and quasi-elliptic otherwise. In the latter instance, the fiber has one singular point, an ordinary cusp; moreover, quasi-elliptic fibrations can occur only in characteristics 2 and 3 [L2, Propositions 1.1 and 1.2]. We say that a numerically elliptic surface is minimal if no fiber contains an irreducible exceptional curve.

2. Curves of canonical type. Let $K$ be the canonical class of a smooth complete surface $S$ and let $D=\sum n_{i} F_{i}$ be a positive sum of reduced irreducible 
curves $F_{i}$ of $S$. Then $D$ is said to be a curve of canonical type $[\mathbf{M u}]$ if $K \cdot F_{i}=$ $D \cdot F_{i}=0$ for each $i$, and indecomposable if, moreover, $D$ is connected and the greatest common divisor of the integers $n_{i}$ is 1 .

LEMMA (2.1). Let $D=\sum n_{i} C_{i}$ be an indecomposable curve of canonical type on a smooth surface $S$, with each $C_{i}$ reduced and irreducible. Then for every divisor $Z=\sum m_{i} C_{i}$ we have $Z^{2} \leq 0$ with equality precisely when $Z$ is a multiple of $D$.

ProOF. This is a special case of the lemma on p. 28 of $[\mathbf{B}-\mathbf{M}]$.

3. Multiple fibers. Let $f: X \rightarrow B$ be a minimal numerically elliptic surface and let $K$ be the canonical class of $X$. Since a nonzero multiple of $K$ is linearly equivalent to a sum of fibers of $X$ [B-M, Theorem 2] it follows that every fiber of $f$ is a curve of canonical type. A fiber $m F$ for which $F$ is indecomposable is said to be of multiplicity $m$ and to be a multiple fiber when $m \geq 2$. The order of the normal bundle $O_{F} \otimes O_{X}(F)$ of $F$ in $X$ divides $m$ [B-M, Proposition 4]. If the order equals $m$, the fiber $m F$ is said to be tame. Otherwise it is said to be wild. A useful fact is

PROPOSITION (3.1). Let $X$ be a minimal numerically elliptic surface with $H^{1}\left(X, O_{X}\right)=0$, as occurs, for example if $X$ is rational. Then any multiple fiber of $X$ is tame.

PROOF. This is Proposition 2.5 of [L2].

4. Rational numerically elliptic surfaces. A rational minimal numerically elliptic surface $f: X \rightarrow B$ enjoys some special properties. By (3.1), any multiple fiber is tame, and since $X$ is rational, so is $B$; i.e., $B$ is isomorphic to the projective line $\mathbb{P}^{1}$. We also have:

PROPOSITION (4.1). Let $f: X \rightarrow B$ be a rational minimal numerically elliptic surface.

(a) Every fiber of $f: X \rightarrow B$ is linearly equivalent to $-m K$, for some fixed positive integer $m$.

(b) The linear system $|-K|$ is not empty.

(c) The fibration $f$ has at most one multiple fiber, and its multiplicity is $m$. There are no multiple fibers precisely in the case that $|-K|$ is a pencil, in which case $f$ has a section.

Proof. Since $B$ is $\mathbb{P}^{1}$, all fibers are linearly equivalent. By the BombieriMumford formula [B-M, Theorem 2] for $K$ we have

$$
K \sim-F+\sum\left(m_{i}-1\right) F_{i}
$$

where $F$ is any fiber and the sum is over the multiple fibers $\left\{m_{i} F_{i}\right\}$ of $f$, the coefficients $m_{i}$ being the multiplicities. In particular, $K \cdot K=0$, and, keeping in mind that $h^{0}(X, 2 K)=0$ for any smooth rational surface $X$ by the birational invariance of the plurigenera [Ha, p. 190], it follows from duality and the RiemannRoch formula that $h^{0}(X,-K)>0$. This proves (b).

Since $K$ is antieffective, we can proceed as on p. 32 of $[\mathbf{B}-\mathbf{M}]$ by intersecting $(*)$ with an ample divisor class to obtain the inequality $-1+\sum\left(1-\left(m_{i}\right)^{-1}\right)<0$. In particular, the summation has at most one term, so by $(*)$ we have $-m K \sim F$, and 
either $m=1$, in which case there are no multiple fibers, or $m>1$, in which case there is precisely one multiple fiber, of multiplicity $m$. Since the general fiber is a multiple $m$ of $-K$, but $K \cdot K=0$, we see $|-m K|$ is composed with a pencil. Since the general fiber is reduced and irreducible, $|-K|$ is a pencil iff $m=1$. Finally, the next lemma shows that $X$ contains an irreducible exceptional curve, and the adjunction formula shows that it must be a section if $|-K|$ is a pencil. This proves (a) and (c).

We have just seen for a rational minimal numerically elliptic surface $X$ that the linear system $\left|-K_{X}\right|$ is not empty and any element is a curve of canonical type, so the next well-known result shows that $X$ is the consecutive blowing-up of the projective plane $\boldsymbol{P}^{2}$ at nine (possibly infinitely near) points.

LEMMA (4.2). Let $S$ be a smooth rational surface having an irreducible curve $F$ linearly equivalent to a positive multiple of $-K_{S}$, and suppose that $9-K_{S} \cdot K_{S} \geq 2$. Then $S$ is obtained by consecutively blowing-up precisely $r=9-K_{S} \cdot K_{S}$ (possibly infinitely near) points of the projective plane $\mathbb{P}^{2}$.

PROOF. This is standard but special enough that we sketch the proof. Any relatively minimal model for a rational surface is either $\mathbb{P}^{2}$ or a Hirzebruch surface $\Sigma_{e}$, which we recall is a $\mathbf{P}^{1}$-bundle over $\mathbb{P}^{1}$ with section $C$ of self-intersection $-e$, $1 \neq e \geq 0$, and we may add that $C$ is never a multiple of the canonical class. Now $S$ is a blowing-up of one of the relatively minimal models. The image to the relatively minimal model of any effective antipluricanonical curve on $S$ is still antipluricanonical, and the only relatively minimal models having an irreducible antipluricanonical curve are $\mathbb{P}^{2}$ and the Hirzebruch surfaces $\Sigma_{e}, e=0,2$. But any blowing-up of $\Sigma_{0}$, and any blowing-up of $\Sigma_{2}$ away from $C$, actually has $\mathbb{P}^{2}$ as a minimal model. And any blowing-up of $\Sigma_{2}$ at a point of $C$ has $\Sigma_{3}$ as a minimal model. Thus any rational surface $S$ which has an effective irreducible antipluricanonical divisor must be either $\Sigma_{e}, e=0,2, \mathbb{P}^{2}$, or a blowing-up of $\mathbb{P}^{2}$. Moreover, we have in each case, respectively, $K_{S} \cdot K_{S}=8,8,9,9-r, r$ being the number of points blown up. The lemma is now clear.

We now give a partial converse to (4.1)(a): an antipluricanonical pencil of canonical type gives rise to a numerically elliptic surface.

LEMMA (4.3). Let $S$ be a smooth rational surface with an anticanonical curve $G$ of canonical type such that, for some integer $m,|m G|$ is positive dimensional. Then, taking $m$ to be the least such integer, $|m G|$ induces a morphism $f: S \rightarrow \mathbb{P}^{1}$ exhibiting $S$ as a minimal numerically elliptic surface.

PrOOF. Since $G^{2}=0$, we will know that $|m G|$ has no base point if it has no fixed components. So let $F$ be the fixed part of the linear system $|m G|$. Then $|m G-F|$ is positive dimensional and has no fixed components, whence $(m G-F)^{2} \geq 0$. Since $m$ is the least integer for which $|m G|$ is positive dimensional we see that $F$ is not a nonzero multiple of $G$. If $F$ is nonzero, it follows by $(2.1)$ that $F^{2}<0$ and so $(m G-F)^{2}<0$, which is a contradiction.

Therefore, $|m G|$ defines a morphism $f: S \rightarrow \mathbb{P}^{1}$. Minimality of $m$ implies that the generic fiber of $f$ is reduced and irreducible, of arithmetic genus 1 by the genus formula. To see that $S$ is numerically elliptic, we only need to show that $f_{*} O_{S}$ is isomorphic to $O_{p_{1}}$. But $f$ is flat since $S$ is irreducible and reduced and dominates 
$\mathbb{P}^{1}$, so $f_{*} O_{S} \cong O_{\mathbf{P}^{1}}$ follows from base change $[\mathrm{Ha}]$ if $h^{0}\left(D, O_{D}\right)=1$ for each fiber $D$ of $f$. By taking cohomology of the exact sequence

$$
0 \rightarrow O_{S}(-D) \rightarrow O_{S} \rightarrow O_{D} \rightarrow 0,
$$

it is clearly enough to show for some (and hence every, since the fibers are all linearly equivalent) fiber $D$ that $h^{1}\left(S, O_{S}(-D)\right)=0$, and this follows if we have $h^{0}\left(D, O_{D}\right)=1$ for any fiber $D$, which we do for any $D$ which is reduced and irreducible. Finally, $S$ is minimal since for any irreducible exceptional curve $E$ we have $E \cdot K=-1$. Thus $E \cdot G=1$, so $E$ cannot be contained in a fiber.

5. Kodaira fiber-types. Kodaira [Ko] classifies all possible connected indecomposable curves of canonical type. We refer to these classes as fiber-types. Those that represent irreducible curves are denoted $\mathrm{I}_{0}, \mathrm{I}_{1}$, and II, representing, respectively, a smooth elliptic curve, a rational curve with a simple node, and a rational curve with a simple cusp. There are also $\mathrm{I}_{2}$, III, and IV, denoting a pair of smooth rational curves taken with multiplicity 1 meeting either transversally at two points $\left(\mathrm{I}_{2}\right)$ or tangentially at one point (III), while IV denotes three smooth rational curves taken with multiplicity 1 which all meet at a single point pairwise transversally. A convenient way to describe the remaining types is via their intersection graphs: each irreducible component, which is always a smooth rational curve when taken with multiplicity 1 , is represented by a node. Two nodes are connected by an edge if the corresponding components meet, which will always be transversally. The graphs, and Kodaira's notation for each, are given in Figure 1. The multiplicities with which each component occurs are indicated for the various types by the numbers which in the figure accompany the nodes; lack of a number means the multiplicity is 1 .

The reader may notice that the graphs are all extended Dynkin diagrams. This suggests using Dynkin diagram terminology for the corresponding fiber-type, as is done in $[\mathrm{RS}]$. Then $\mathrm{I}_{n}, n \geq 1$, becomes $A_{n-1} ; \mathrm{II}^{*}, \mathrm{III}^{*}$, and $\mathrm{IV}^{*}$ become $E_{8}, E_{7}$, and $E_{6}$; and $I_{n}^{*}, n \geq 0$, becomes $D_{n+4}$. For completeness, II, III and IV become $A_{0}^{*}, A_{1}^{*}$, and $A_{2}^{*}$. It also seems reasonable to denote $\mathrm{I}_{0}$ by $A_{-1}$. However, not all of these types will concern us here.

PROPOSITION (5.1). A connected curve of canonical type on a rational minimal numerically elliptic surface $X$ has at most nine irreducible components.

ProOF. Suppose $D$ is a connected indecomposable curve of canonical type, and write $D$ as a sum $\sum n_{i} C_{i}$ of multiples of reduced irreducible curves $C_{i}$. If we show that the components $\left\{C_{i}\right\}$ represent linearly independent elements of Pic $X \otimes \mathbb{Q}=$ $V$, then the result follows since $\operatorname{dim}_{\mathbb{Q}} V=10$ (cf. (4.2)) and the components $\left\{C_{i}\right\}$ lie in the subspace perpendicular to the canonical class $K_{X}$, and therefore span a subspace of $V$ of dimension no more than 9 .

So suppose there are integers $\left\{m_{i}\right\}$ such that $F=\sum m_{i} C_{i}$ is linearly equivalent to 0 . Then $F^{2}=0$ and we see that $F$ is a multiple of $D$ by (2.1). Since Pic $X$ has no torsion, $F$ must be the zero multiple of $D$, whence $m_{i}=0$ for all $i$, proving the components $\left\{C_{i}\right\}$ are indeed linearly independent.

The Halphen transformation is the means by which we will construct multiple fibers. To define it we require some knowledge of the group $\mathrm{Pic}^{0} F$ of divisor classes of $F$ whose restriction to each component of $F$ has degree zero. 

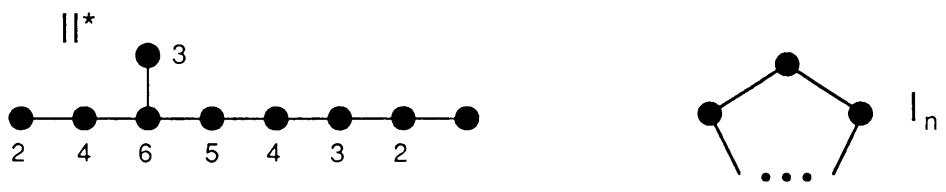

a cycle of $n \geq 3$ nodes
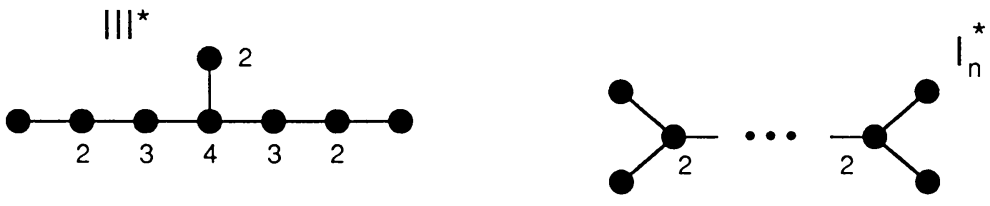

$n+5$ nodes, $n \geq 1$
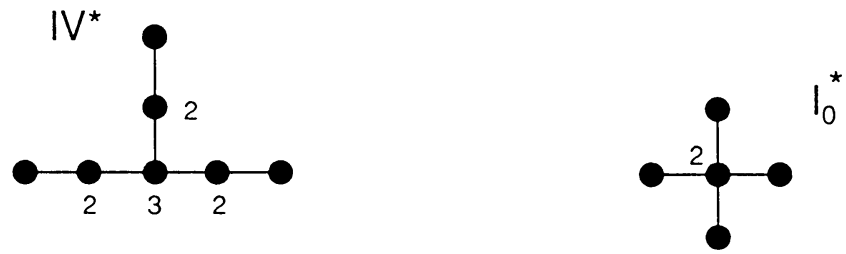

FIGURE 1

PROPOSITION (5.2). Let $F$ be an indecomposable curve of canonical type, let $q$ be a smooth closed point of $F$ and let $F_{q}^{0}$ denote the connected component of smooth points of $F$ which contains $q$. Then there is a natural bijection $\mathrm{Pic}^{0} F \rightarrow F_{q}^{0}$ taking 0 to $q$. Moreover, $\mathrm{Pic}^{0} F$ is isomorphic to the multiplicative group $\mathbf{G}_{m}$ of units of the ground field $k$ if $F$ has type $\mathrm{I}_{n}, n \geq 1$. For $F$ of any remaining type except $\mathrm{I}_{0}$, $\mathrm{Pic}^{0} F$ is isomorphic to the additive group $\mathbf{G}_{a}$ of $k$.

PROOF. For the proof of the first statement, see the proof of Theorem 2.6 on p. 288 of $[\mathbf{D R}]$; indeed, $\mathrm{Pic}^{0} F$ is represented by the scheme $F_{q}^{0}$. But $F_{q}^{0}$ is the punctured affine line (and hence the group structure must be $\mathbf{G}_{m}$ ) in the cases $\mathrm{I}_{n}$, $n \geq 1$, and $F_{q}^{0}$ is the affine line (and hence must be $\mathbf{G}_{a}$ ) for the remaining cases except $\mathrm{I}_{0}$.

Note (5.3). The preceding proposition justifies referring to a fiber-type $\mathrm{I}_{n}, n \geq 1$, as being of multiplicative type and to the others excepting $\mathrm{I}_{0}$ as being of additive type.

6. Fibers on surfaces with section. As grist for the mill of Proposition (7.1), we here note the well-known fact that (5.1) has the following partial converse.

PROPOSITION (6.1). Every Kodaira type with nine or fewer components occurs as the type of a fiber on some rational minimal elliptic surface with section.

PROOF. The proof is merely to construct for each type an example of such a surface with a fiber of that type, which constructions we sketch for the convenience of the reader. The types having three or fewer components, i.e., $\mathrm{I}_{0}, \mathrm{I}_{1}, \mathrm{I}_{2}, \mathrm{I}_{3}, \mathrm{II}$, III, or IV, occur as cubic curves in $\mathbb{P}^{2}$. Suppose for a cubic curve $D$ of one of these types one takes a smooth cubic curve $C$ meeting $D$ transversally and blows up the points of intersection, obtaining a surface $X$. The proper transforms of $C$ and $D$ are curves of canonical type, obviously moving in a pencil. Hence by (4.3), 
$X$ is a minimal elliptic surface the fibers of which are the proper transforms of the elements of the cubic pencil defined by $C$ and $D$. In particular, a fiber isomorphic to $D$ occurs. And since the fibers are anticanonical, there is by $(4.1)(c)$ no multiple fiber; indeed, any irreducible exceptional curve is a section.

Let us now see that fibers of type $\mathrm{I}_{4}$ can occur. Let $x_{i}, i=0,1,2$, be homogeneous coordinates on $\mathbb{P}^{2}, D_{i}, i=0,1,2$, the corresponding coordinate lines. Let $D$ now be the divisor $D_{0}+D_{1}+D_{2}$, and let $C$ now be a smooth cubic curve meeting each component of $D$ transversally and passing through precisely one singular point of $D$, say $x_{0}=0=x_{1}$. Blow up the points of intersection of $C$ and $D$, denoting proper transforms with a prime $\left({ }^{\prime}\right)$. Also denote the blowing-up of the point $x_{0}=0=x_{1}$ by $E$. It is easy to check that $D^{\prime}+E$ and $C^{\prime}$ are anticanonical, meeting transversally at a point $q$ of $E$. Blow up $q$ and denote the resulting surface by $X$. Then $D^{\prime \prime}+E^{\prime}$ and $C^{\prime \prime}$ are anticanonical and in fact of canonical type on $X$. As above, it follows that $X$ is a rational minimal elliptic surface with section having $D^{\prime \prime}+E^{\prime}$ as a fiber, which it is easy to check is of type $\mathrm{I}_{4}$.

That the other fiber-types with nine or fewer components also occur can be seen in a similar way. For each of the remaining types we give a pair of cubic curves, $C$ a smooth one and $D$ a reducible one, and we describe how they meet. We leave it to the reader to check that the plane pencil determined by $C$ and $D$ corresponds, as above, to a minimal elliptic surface with section, the fiber corresponding to $D$ having the desired fiber-type. We again take plane homogeneous coordinates $x_{i}$, $i=0,1,2$, and denote the coordinate lines by $D_{i}, i=0,1,2$. For distinct elements $i, j, k$ of $\{0,1,2\}$, denote by $q_{k}$ the point of intersection of $D_{i}$ and $D_{j}$.

For a fiber of type $\mathrm{I}_{n}, n=5,6,7,8$, or 9 , take $D$ to be the divisor $D_{0}+D_{1}+D_{2}$. For $n=5$ or 6 , take $C$ meeting each component of $D$ transversally and passing through precisely $n-3$ singular points of $D$. For $n=7,8$, or 9 , take $C$ to be any smooth cubic curve passing through the three singular points of $D$ such that wherever else $D$ and $C$ meet, they meet transversally. Moreover, for $n=7$, require $C$ to be tangent to $D_{1}$ at $q_{2}$, and to meet each component of $D$ transversally otherwise; for $n=8$, require $C$ to be tangent to $D_{1}$ at $q_{2}$, to be tangent to $D_{2}$ at $q_{0}$, and to meet each component of $D$ transversally otherwise; and for $n=9$, require $C$ to be tangent to $D_{1}$ at $q_{2}$, to be tangent to $D_{2}$ at $q_{0}$, and to be tangent to $D_{0}$ at $q_{1}$. If $\operatorname{char}(k)=3$, we note that for the conditions of case $n=9$ to be met, it is merely necessary that $C$ not be supersingular.

For II $^{*}$, take $D$ to be the divisor $3 D_{0}$ and $C$ to have a flex at $q_{2}$ and to be tangent there to $D_{0}$. For III*, take $D$ to be the divisor $3 D_{0}$ and $C$ to pass through $q_{1}$ and to be tangent at $q_{2}$ to $D_{0}$. For $\mathrm{IV}^{*}$, take $D$ to be the divisor $3 D_{0}$ and $C$ to meet $D_{0}$ transversally.

There only remain the cases $\mathrm{I}_{n}^{*}, n=0, \ldots, 4$. Take $D$ to be the divisor $2 D_{2}+D_{1}$. For $n=0$, take $C$ to meet $D_{2}$ and $D_{1}$ transversally and to not pass through $q_{0}$. For $n=1$, take $C$ to meet $D_{2}$ and $D_{1}$ transversally and to pass through $q_{0}$. For $n=2$, take $C$ to meet $D_{2}$ transversally, to pass through $q_{0}$ tangent to $D_{1}$, and to pass through $q_{2}$. For $n=3$, take $C$ to meet $D_{2}$ transversally, to pass through $q_{0}$ tangent to $D_{1}$, and to have a flex at $q_{0}$. Finally, for $n=4$, take $C$ to be tangent to $D_{2}$ at $q_{1}$, to pass through $q_{0}$ tangent to $D_{1}$, and to have a flex at $q_{0}$. This last case requires that $C$ not be supersingular if the characteristic is 2 , since $\operatorname{Pic}^{0} C$ must have nontrivial 2 -torsion. 
7. The Halphen transform. We define here what we call a Halphen transformation, a birational transformation of a rational numerically elliptic surface. Let $X^{\prime}$ be a rational minimal numerically elliptic surface and let $m^{\prime} F^{\prime}$ be a fiber of multiplicity $m^{\prime}$ such that $F^{\prime}$ is anticanonical. By (4.2), $X^{\prime}$ has an irreducible exceptional curve; let $E$ be any such curve. Then by the adjunction formula we have $E \cdot F^{\prime}=1$; in particular, $E$ meets $F^{\prime}$ at a smooth point $q$ of $F^{\prime}$ on a reduced component $F_{q}$ of $F^{\prime}$.

Now let $L$ be a divisor class of finite order, say $m$, in $\operatorname{Pic}^{0} F$. By (5.2), there is a unique smooth point $t$ of $F^{\prime}$ on the component $F_{q}$ such that $O_{F}(q-t)$ is isomorphic to $O_{X^{\prime}}\left(-F^{\prime}\right) \otimes L$. Define the Halphen transform of $X^{\prime}$ associated to the pair $(E, L)$ to be the surface $X$ obtained by contracting $E$ and blowing up the image of $t$ under the contraction. We refer to the birational transformation $X^{\prime} \rightarrow X$ as a Halphen transformation. Clearly, $F^{\prime}$ is canonically isomorphic under the Halphen transformation to its proper transform, which we denote by $F$, and, as we will see, both are anticanonical divisors. The utility to us of the Halphen transformation depends on the next result.

PROPOSITION (7.1). The Halphen transform $X$ of $X^{\prime}$ associated to $(E, L)$ is a rational minimal numerically elliptic surface. Nioreover, $L$ is isomorphic (under the canonical identification of $F^{\prime}$ with $F$ ) to the normal bundle $O_{X}(F) \otimes O_{F}$ of $F$ in $X$ and $m F$ is a fiber of the numerically elliptic fibration on $X$ of multiplicity $m$, $m$ being the order of $L$ in $\mathrm{Pic}^{0} F$.

PROOF. In the notation of the paragraph preceding the proposition, if $t=q$, then $L$ and $O_{X}(F) \otimes O_{F}=O_{X^{\prime}}\left(F^{\prime}\right) \otimes O_{F^{\prime}}=O_{F^{\prime}}\left(F^{\prime}\right)$ are isomorphic. In particular, $m=m^{\prime}, X=X^{\prime}$, and the conclusion is clear. So say $t$ and $q$ are distinct.

Take $Y$ to be the surface obtained by contracting $E$ to a point. It is convenient to also denote by $q$ and $t$ the images of $q$ and $t$ under the contraction of $E$. Then $X$ is the blowing-up of $Y$ at $t$. Denote the blowing-up of $X^{\prime}$ at $t$ by $X^{\prime \prime}$, and the total transform of $t$ on $X^{\prime \prime}$ by $E_{t}$. We now have the blowing-up on $X^{\prime}$ of $t, \pi^{\prime}: X^{\prime \prime} \rightarrow X^{\prime}$, and the blowing-up on $X$ of $q, \pi: X^{\prime \prime} \rightarrow X$. These induce natural maps on the Picard groups, which are injective [Ha]. Denoting the proper transform of $F^{\prime}$ on $X^{\prime \prime}$ by $F^{\prime \prime}$, we see that $\pi^{*}(F)$ is linearly equivalent to $F^{\prime \prime}+\left(\pi^{\prime}\right)^{*}(E)$ and that $F^{\prime \prime}+E_{t}$ is linearly equivalent to $\left(\pi^{\prime}\right)^{*}\left(F^{\prime}\right)$. But for any divisor class $D$ of $X$ it is clear that $D \otimes O_{F}$ is isomorphic, with respect to the canonical identification of $F$ with $F^{\prime \prime}$, to $\left(\pi^{*} D\right) \otimes O_{F}$, and similarly for $X^{\prime}$. Applying this principle repeatedly, we see that the following classes are all isomorphic: $O_{X}(F) \otimes O_{F}, O_{X^{\prime \prime}}\left(F^{\prime \prime}+\left(\pi^{\prime}\right)^{*}(E)\right) \otimes O_{F}$, $O_{X^{\prime \prime}}\left(F^{\prime \prime}\right) \otimes O_{F}(q), O_{X^{\prime \prime}}\left(F^{\prime \prime}+E_{t}\right) \otimes O_{F}(q-t), O_{X^{\prime}}\left(F^{\prime}\right) \otimes O_{F}(q-t), O_{X^{\prime}}\left(F^{\prime}\right) \otimes$ $O_{X^{\prime}}\left(-F^{\prime}\right) \otimes L$, and $L$. In particular, the normal bundle of $F$ on $X$ is isomorphic to $L$, as claimed.

We also see that $F$ is anticanonical on $X: F^{\prime}$ is anticanonical on $X^{\prime}$, whence $\left(\pi^{\prime}\right)^{*}\left(F^{\prime}\right)-E_{t}$ and thus $F^{\prime \prime}$ are linearly equivalent to $-K_{X^{\prime \prime}}$. Likewise, $-K_{X^{\prime \prime}}$ and thus $F^{\prime \prime}$ and $\pi^{*}(F)-\left(\pi^{\prime}\right)^{*}(E)$ are linearly equivalent to $\pi^{*}\left(-K_{X}\right)-\left(\pi^{\prime}\right)^{*}(E)$. But $\pi^{*}$ is injective, so it follows that $F$ is linearly equivalent to $-K_{X}$.

We can now show that the linear system $|m F|$ on $X$ induces a morphism $X \rightarrow \mathbb{P}^{1}$ which exhibits $X$ as a rational minimal numerically elliptic surface having fiber $m F$ of multiplicity $m$. Since $O_{F}(F)=O_{X}(F) \otimes O_{F}$ is isomorphic to $L$, it has order $m$ 
in Pic $F$. It follows by induction on $r$ from the exact sequence

$$
0 \rightarrow O_{X}((r-1) F) \rightarrow O_{X}(r F) \rightarrow O_{F}(r F) \rightarrow 0
$$

(using pp. 332-333 of [Mu] to compute $h^{i}\left(F, O_{F}(r F)\right)$ ) that $m F$ is the least multiple of $F$ for which $|m F|$ is positive dimensional on $X$. The desired morphism $X \rightarrow \mathbb{P}^{1}$ thus exists by (4.3); clearly $m F$ is a fiber. Since $F$ is anticanonical (4.1) proves its multiplicity is $m$.

By taking account of what torsion there is in the Picard group of an indecomposable curve of canonical type and keeping in mind that the notion of a numerically elliptic surface coincides with that of an elliptic surface except in characteristics 2 and 3, Theorem (0.1) follows easily from this next corollary-for characteristics other than 2 or 3 . To obtain a proof in all characteristics more work is required, which we begin with a study of the properties of Halphen transformations following the corollary.

COROLlaRY (7.2). Let $m>1$ be an integer and $T$ a Kodaira fiber-type. Then there exists a rational minimal numerically elliptic surface having a multiple fiber of type $m T$ if and only if there exists a rational minimal numerically elliptic surface with section (i.e., with no multiple fibers (cf. (4.1)(c))) having a fiber $F$ of type $T$ such that $\mathrm{Pic}^{0} F$ has an element of order $m$.

PROOF. Say there is a rational minimal numerically elliptic surface $X$ having a multiple fiber $m F$ of multiplicity $m$ such that $F$ has type $T$. Choose $E$ to be any irreducible exceptional curve (one of which must exist by (4.2)) and $L$ to be the identity element of $\mathrm{Pic}^{0} F$. Then the Halphen transform $X^{\prime}$ of $X$ associated to the pair $(E, L)$ is by $(7.1)$ a rational minimal numerically elliptic surface for which $F$ is an anticanonical fiber. In particular, $X^{\prime}$ is a numerically elliptic surface with section.

Conversely, say $Y^{\prime}$ is a rational minimal numerically elliptic surface with section having a fiber $F$ of type $T$, and say $L$ now is an element of $\operatorname{Pic}^{0} F$ of order $m$. Then by (7.1) $m F$ is a multiple fiber of multiplicity $m$ on the Halphen transform $Y$ of $Y^{\prime}$ associated to the pair $(E, L)$ for any irreducible exceptional curve $E$ of $Y^{\prime}$.

While the Halphen transformation of (7.1) preserves the fiber $F$ it is useful to understand what becomes of other fibers, especially reducible ones. Recall that a smooth rational curve of self-intersection -2 is called a $(-2)$-curve. It follows by the adjunction formula that a reduced irreducible curve is a component of a reducible fiber on a minimal numerically elliptic surface if and only if the curve is a (-2)-curve. Let $X$ be such a surface; a useful datum is the rank $\eta(X)$ of the span in $\operatorname{Pic}(X)$ of the classes of the $(-2)$-curves of $X$. Other useful data come from considering the intersection graphs of the reducible fibers of $X$.

Now consider a rational minimal numerically elliptic surface with section $X^{\prime}$ having an anticanonical fiber $F$ of additive type, and suppose the characteristic of the ground field $k$ is $p$. Let $E^{\prime}$ be an irreducible exceptional curve on $X^{\prime}$. If $p$ is positive, then, since $F$ is of additive type, $\operatorname{Pic}^{0}(F)$ is pure $p$-torsion and for any element $L$ of $\operatorname{Pic}^{0}(F)$ we have the Halphen transform $X_{L}$ of $X^{\prime}$ associated to $\left(E^{\prime}, L\right)$. 
PROPOSITION (7.3). Suppose that $p$ is 2 or 3 and that $L$ is a general element of $\operatorname{Pic}^{0}(F)$. Then we have $\eta\left(X_{L}\right)=\eta\left(X^{\prime}\right)$ and, moreover, there is a 1-1 correspondence between the reducible fibers of $X^{\prime}$ and those of $X_{L}$, and corresponding fibers have the same intersection graph.

We remark that the proof defines precisely which elements $L$ comprise the general set for which the proposition holds. To give the proof, we first need to prove a lemma.

LEMMA (7.4). Let $Y$ be a rational minimal numerically elliptic surface and denote the number of (-2)-curves on $Y$ by $n$ and the number of reducible fibers by $r$. Then we have the formula $\eta(Y)+r=n+1$.

PROOF. For each reducible fiber of $Y$ choose a $(-2)$-curve occurring as a component of that fiber; call these curves marked. Then the span in $\operatorname{Pic}(Y)$ of the (classes of) unmarked (-2)-curves are linearly independent. To see this, suppose that some linear combination of unmarked (-2)-curves were (linearly equivalent to) zero. By grouping together curves coming from the same fiber we obtain elements $v_{i}$ and the aforementioned linear combination becomes $\sum v_{i}=0$. But distinct summands come from distinct fibers and so are perpendicular. Thus we see that $0=\left(\sum v_{i}\right)^{2}=\sum\left(v_{i}\right)^{2}$. But by $(2.1)$ we know that $\left(v_{i}\right)^{2}<0$ for any nonzero sum $v_{i}$ of unmarked $(-2)$-curves coming from a single fiber. It follows that $v_{i}$ is the zero sum and hence that the unmarked $(-2)$-curves are linearly independent.

On the other hand, any marked (-2)-curve is (linearly equivalent to) a rational linear combination of the canonical class $K_{Y}$ and a sum of unmarked (-2)-curves. Since $K_{Y}$ lies in the rational span of unmarked $(-2)$-curves together with any marked (-2)-curve, it follows that the rank of the span of the $(-2)$-curves equals $n-r+1$, the rank of the span of the unmarked (-2)-curves plus 1 for any marked (-2)-curve; i.e., $\eta(Y)=n-r+1$.

PROOF OF (7.3). By definition, the Halphen transformation $X^{\prime} \rightarrow X_{L}$ factors as $X^{\prime} \rightarrow X^{\prime \prime} \leftarrow X_{L}$, where the first morphism is the contraction of $E^{\prime}$ and the second morphism is the blowing-up of (the image of) the point $t$ of $F$ for which $O_{X^{\prime}}\left(E^{\prime}\right) \otimes O_{F}(-t)$ is isomorphic to $L$. Now the $\operatorname{rank}$ of $\operatorname{Pic}\left(X^{\prime \prime}\right)$ is 9 , so $X^{\prime \prime}$ has only finitely many irreducible exceptional curves $[\mathbf{M}]$, so it is sensible to speak of the Zariski-open subset $U$ of smooth points of $F$ which lie on the component of $F$ containing $t$ but on no other irreducible exceptional curve of $X^{\prime \prime}$.

I claim that if $L$ is chosen so that $t$ lies in $U$, then the blowing-up $E_{L}$ of the point $t$ in $X^{\prime \prime}$ meets precisely one component of each reducible fiber of $X_{L}$ and for a nonmultiple fiber this component has multiplicity one. To see this, suppose (suppressing the subscript $L$ ) that $E$ were to meet two or more components of some nonmultiple reducible fiber $G$. Now $G$, being a fiber, is equal to $-m K_{X}$, where $m$ is $p$ (and so either 2 or 3 ) except in the case that $L$ is trivial and $m=1$. But $E \cdot G=m$ by adjunction and since, in any case, we have $m \leq 3$, we see that $E$ would meet some component (which we denote $N$ ) of $G$ precisely once. Because $N$ is a (-2)-curve, its image in $X^{\prime \prime}$ under the contraction of $E$ would be an irreducible exceptional curve containing $t$; i.e., $t$ would not lie in $U$, contrary to hypothesis. Thus we may assume that $E$ meets precisely one component of each reducible fiber. Now suppose $E$ were to meet a nonreduced component (of multiplicity $\mu$, say) of some nonmultiple reducible fiber $G$. We may denote this component $\mu M$, and again 
$M$ is a (-2)-curve. Since $m=E \cdot G=E \cdot \mu M$ still holds and $m$ is a multiple of $\mu$, this is impossible if $m=1$, so it would have to be that $m=p, p$ being 2 or 3 . Thus $m=\mu=p$ and $E \cdot M=1$; as before $E$ meets a (-2)-curve transversally, which leads to a contradiction.

Having finished the proof of our claim, note that any (-2)-curve on $X^{\prime}$ that does not meet $E^{\prime}$ survives through the Halphen transformation to become a $(-2)$-curve on $X$ (we continue to suppress subscripts), and conversely any (-2)-curve on $X$ that does not meet $E$ must have come via the Halphen transformation from a (-2)curve on $X^{\prime}$. This establishes a bijective correspondence $\beta$ between the $(-2)$-curves of $X^{\prime}$ that do not meet $E^{\prime}$ and those of $X$ that do not meet $E$.

Now both $E^{\prime}$ (because it is a section of the fibration on $X^{\prime}$ ) and $E$ (by our claim) meet precisely one component of each reducible fiber on their respective surfaces $X^{\prime}$ and $X$. Since every reducible fiber has at least two components, $\beta$ establishes a bijective correspondence between the reducible fibers of $X^{\prime}$ and $X$, so we see that both surfaces have equal numbers of reducible fibers and equal numbers of $(-2)$-curves. It follows by (7.4) that $\eta\left(X_{L}\right)=\eta\left(X^{\prime}\right)$. Moreover, an examination of the intersection graphs of the reducible Kodaira fiber-types shows to be distinct the graphs obtained by deleting any vertex corresponding to a component of multiplicity one. Hence corresponding reducible fibers of $X^{\prime}$ and $X$ have the same intersection graphs.

8. Quasi-elliptic fibrations. We now set down some properties of quasielliptic fibrations on rational surfaces, quite similar to what is done for $K 3$-surfaces on pp. 150-151 of [RS]. It is convenient to introduce some terminology: the weight of a fiber of multiplicity $m$ of a numerically elliptic fibration will denote the number of components of multiplicity $m$ of the fiber. The following proposition is well known but hard to reference.

PROPOSITION (8.1). Let $X$ be a rational minimal numerically elliptic fibration.

(i) If $X$ is quasi-elliptic, all fibers of $X$ have additive type.

(ii) If $X$ is quasi-elliptic, the rank $\eta(X)$ of the span in Pic $X$ of the (-2)-curves of $X$ is 9 .

(iii) If $\eta(X)=9$, then the product of the weights of all of the fibers is an integer squared, and if, moreover, $X$ is quasi-elliptic, then the weight of any fiber is a power of the characteristic $p$.

(iv) If $\eta(X)=9$ and if $X$ has a section, then the number of irreducible exceptional curves on $X$ is the square root of the product of the weights of the fibers.

The proof depends on some notions that we now recall. Let $l$ be a prime number and denote by $\mathbb{Q}_{l}$ the field of $l$-adic rational numbers. For a variety $Y$ over a separably closed field one defines the l-adic Betti numbers to be

$$
\beta_{r}(Y, l)=\operatorname{dim}_{\mathbb{Q}_{l}} H^{r}\left(Y_{e t}, \mathbb{Q}_{l}\right)
$$

and then the $l$-adic Euler-Poincaré characteristic is

$$
\chi\left(Y_{e t}, l\right)=\sum(-1)^{r} \beta_{r}(Y, l) ;
$$

see [Mi, p. 166]. 
LEMMA (8.2). Let $Y$ be a normal surface and let $f: Y \rightarrow C$ be a flat proper morphism over a smooth curve $C$ such that $f_{*}\left(O_{Y}\right) \cong O_{C}$. Let $Y_{y}$ and $Y_{x}$ be the fibers of $f$ over, respectively, a closed point $y$ and a geometric generic point $x$ of $C$. Then, for a prime number l relatively prime to char $(k)$, we have: $\chi\left(Y_{y}, l\right) \geq \chi\left(Y_{x}, l\right)$ and $\beta_{1}\left(Y_{y}, l\right) \leq \beta_{1}\left(Y_{x}, l\right)$.

PROOF. In case $Y$ is smooth, the proof of Lemma 1 of [L3] gives a different argument for this. We proceed by showing that the Betti numbers $\beta_{0}$ and $\beta_{2}$ are upper-semicontinuous functions of the fibers, while $\beta_{1}$ is lower-semicontinuous. This gives the result.

Let $\mathbf{F}$ be the field $\mathbb{Z} / l \mathbb{Z}$. The groups $H^{i}\left(Y_{t}, \mathbf{F}\right), t=y, x$, are finite (Corollary VI.2.8 [Mi]) so we can compute Euler characteristics with $\mathbf{F}$ in place of $\mathbb{Q}_{l}$ [Mi, p. 166]. Since $f_{*}\left(O_{Y}\right) \cong O_{C}$, the fibers of $f$ are connected (cf. [Ha, p. $279])$, whence $\operatorname{dim}_{\mathbf{F}} H^{0}\left(Y_{y}, \mathbf{F}\right)=\operatorname{dim}_{\mathbf{F}} H^{0}\left(Y_{x}, \mathbf{F}\right)=1$. Thus $\beta_{0}$ is constant on the fibers. Since $Y$ is a normal surface it has at most finitely many singularities, whence $Y_{x}$ is an irreducible curve. But $H^{2}$ measures the number of irreducible components of a curve (cf. Lemma VI.11.3, Remark V.2.4a and p. 163 of [Mi]) so $1=\operatorname{dim}_{\mathbf{F}} H^{2}\left(Y_{x}, \mathbf{F}\right) \leq \operatorname{dim}_{\mathbf{F}} H^{2}\left(Y_{y}, \mathbf{F}\right)$, so $\beta_{2}$ is upper-semicontinuous.

Finally, consider $\beta_{1}$. By Proposition 6.3.5 together with example 6.1.6 of [R] we have a specialization map of fundamental groups $\pi_{1}\left(Y_{x}\right) \rightarrow \pi_{1}\left(Y_{y}\right)$ and the cokernel. is finite. Applying $\operatorname{Hom}(\cdot, \mathbf{F})$ we obtain a homomorphism

$$
\operatorname{Hom}\left(\pi_{1}\left(Y_{y}\right), \mathbf{F}\right)=H^{1}\left(Y_{y}, \mathbf{F}\right) \rightarrow H^{1}\left(Y_{x}, \mathbf{F}\right)=\operatorname{Hom}\left(\pi_{1}\left(Y_{x}\right), \mathbf{F}\right)
$$

which is injective for all but finitely many $l$. Thus $\beta_{1}$ is lower-semicontinuous for almost all $l$. But we have seen that $\beta_{0}$ and $\beta_{2}$ are independent of $l$ different from $\operatorname{char}(k)$, and by [Do] this is true of $\chi$, so it is also true of $\beta_{1}$. Thus $\beta_{1}$ is lowersemicontinuous for any $l$ different from $\operatorname{char}(k)$.

PROOF OF $(8.1)$. (i) Note that by $(8.2), \beta_{1}$ is lower-semicontinuous, while the generic fiber of a quasi-elliptic fibration, being of additive type, has $\beta_{1}=0$. Therefore, every fiber has $\beta_{1}=0$, but the only Kodaira types with $\beta_{1}=0$ are the ones of additive type.

(ii) We have a natural map $\pi: \operatorname{Pic} X \rightarrow \operatorname{Pic} C$, where $C$ is the generic fiber. Now $\pi^{-1}\left(\mathrm{Pic}^{0} C\right)$ is $K^{\perp}$, the subgroup of Pic $X$ perpendicular to the canonical class $K$ of $X$. Thus $K^{\perp}$ has rank 9 and it contains the kernel $J$ of the natural map $\pi:$ Pic $X \rightarrow \operatorname{Pic} C$. However, $\mathrm{Pic}^{0} C$ is pure $p$-torsion since $C$ is of additive type, so $J$ also has rank 9 . But it is not hard to see $J$ is generated by the components of fibers, that is, by $-K$ and by the $(-2)$-curves. Therefore there must be $(-2)$ curves, and hence there occur reducible fibers. But since a reducible fiber is both a multiple of $K$ and a sum of $(-2)$-curves, the span $L$ of the (-2)-curves must have rank 9, the rank of $J$. Moreover, the order of $K^{\perp} / L$ is a power of $p$. To see this, note that $K^{\perp} / J$ has $p$-power order while $J$ is generated by $L$ and $K$. Since $\eta(X)=9, X$ has a reducible fiber and this fiber is linearly equivalent to $-p K$, so $-p K$ is in $L$. Thus multiplication by $p$ annihilates $J / L$, so it has $p$-power order, and thus so must $K^{\perp} / L$.

(iii) Let $L$ be the span of the $(-2)$-curves and consider the restriction of the intersection form of Pic $X$ to $K^{\perp}$. Its radical is generated by the canonical class $K$; i.e., any isotropic vector is a multiple of $K$. It follows that the quotients

$$
L^{-}=L /(L \cap \mathbb{Z} K) \subset\left(K^{\perp}\right)^{\wedge}=K^{\perp} / \mathbb{Z} K
$$


are nondegenerate and free as $\mathbb{Z}$-modules. But $\eta(X)=9$, so both have rank 9 . Thus there is a $\mathbb{Z}$-module isomorphism $A:\left(K^{\perp}\right)^{\wedge} \rightarrow L^{\wedge}$, so the order of $\left(K^{\perp}\right)^{\curlyvee} / L^{\wedge} \cong$ $K^{\perp} /(L+\mathbb{Z} K)$ equals $|\operatorname{det}(A)|$. If $Q$ is the intersection form on $\left(K^{\perp}\right)$, then the determinant of the intersection form $Q_{L^{\wedge}}$ on $L^{\wedge}$ is $\operatorname{det}\left(A^{t} Q A\right)$. But $\operatorname{det}(Q)=1$ since $\left(K^{\perp}\right)^{\wedge}$ is unimodular, so $\operatorname{det}\left(Q_{L^{\wedge}}\right)=\operatorname{det}(A)^{2}$. Since $K^{\perp} / L$ has $p$-power order if $X$ is quasi-elliptic, its quotient $\left(K^{\perp}\right)^{\mathcal{Y}} / L^{\wedge}$ has order a power of $p$. So not only is $\operatorname{det}\left(Q_{L^{\wedge}}\right)$ a square, but, if $X$ is quasi-elliptic, it is a power of $p$, too.

Since $L$ is generated by $(-2)$-curves (the components of reducible fibers), $L^{\wedge}$ is the direct sum $\bigoplus\left(L_{i}\right)$, where the sum is over all reducible fibers, $L_{i}$ is the span of the (-2)-curves of the given fiber, and $\left(L_{i}\right)^{-}$is the quotient $L_{i} /\left(L_{i} \cap \mathbb{Z} K\right)$. This sum is compatible with the intersection form $Q_{L^{\wedge}}$, so $Q_{L^{\wedge}}$ is a direct sum $\bigoplus\left(Q_{L_{i}^{\hat{i}}}\right)$ of the intersection forms $Q_{L_{\hat{i}}}$ induced by each reducible fiber. Hence, $\operatorname{det}\left(Q_{L^{\wedge}}\right)=\prod \operatorname{det}\left(Q_{L_{\hat{i}}^{\hat{i}}}\right)$, and it is easy to check that $\pm \operatorname{det}\left(Q_{L_{\hat{i}}}\right)$ in each case equals the weight of the fiber. (Indeed, the intersection graph of each reducible fiber is the extended Dynkin diagram of the Dynkin diagram corresponding to a finite dimensional complex Lie algebra, the determinant of the Cartan matrix of which is $\pm \operatorname{det}\left(Q_{L_{i}^{\hat{i}}}\right)$.)

Since the weight of an irreducible fiber is 1 , it follows that the product of the weights of the fibers is the product of the weights of the reducible fibers, which we thus see is a square and, if $X$ is quasi-elliptic, a power of $p$.

(iv) If $X$ has a section, then $K \in L=J$, so $\left(K^{\perp}\right)^{\wedge} / L^{\wedge} \cong K^{\perp} / J$. Thus, as we saw above, $K^{\perp} / J$ has order the square root of the product of the weights of the fibers. But the irreducible exceptional curves are precisely the sections of the numerically elliptic fibration, and these are precisely the points of the generic fiber $C$ defined over the function field $k\left(\mathbb{P}^{1}\right)$ of the base curve of the fibration. Thus an element of $\mathrm{Pic}^{0} C$ defined over $k\left(\mathbb{P}^{1}\right)$ is the restriction to $C$ of the difference of two exceptional curves, so the order of the $k\left(\mathbf{P}^{1}\right)$-points of $\mathrm{Pic}^{0} C$ is just the number of exceptional curves. But the difference of two exceptional curves lies in $K^{\perp}$; in particular, $K^{\perp} / J$ surjects onto $\mathrm{Pic}^{0} C$, whence $K^{\perp} / J$ is isomorphic to $\mathrm{Pic}^{0} C$, and the conclusion follows.

We now want to study when we may guarantee that the Halphen transform of a rational minimal elliptic surface $X$ with section is again elliptic. Say $F$ is the fiber of $X$ that becomes multiple under the Halphen transformation. The Halphen transform will be elliptic either if $F$ is not of additive type (by (8.1)(i)), or if the characteristic $p$ is not 2 or 3 (by $\S 1$ ), so, to study the interesting case, suppose that $F$ is of additive type and that $p$ is 2 or 3 . Let $E$ be an irreducible exceptional curve of $X$, and denote by $X_{L}$ the Halphen transform of $X$ associated to $(E, L)$, where $L$ is an element of $\operatorname{Pic}^{0} F$. Note that $X_{L}$ is defined for any element $L$ of $\operatorname{Pic}^{0} F$ since $F$ has additive type and thus $\operatorname{Pic}^{0} F$ is pure $p$-torsion. We can now state:

PROPOSITION (8.3). In the situation of the preceding paragraph, suppose that $X_{L}$ is quasi-elliptic for a general element $L$. Then one of the two cases below must hold:

(i) $F$ is the only reducible fiber of $X$, and either $p=3$ and $F$ has type $E_{8}$, or $p=2$ and $F$ has type $E_{8}$ or $D_{8}$; or 
(ii) $F$ is one of only two reducible fibers of $X$, and (denoting the other reducible fiber by $G$ ) either $p=3, F$ has type $E_{6}$, and $G$ has type $A_{2}$, or $p=2, F$ has type $E_{7}$, and $G$ has type $A_{1}$.

REMARK (8.4). Note that we are not saying that $X_{L}$ will be quasi-elliptic for a general $L$ in the cases enumerated in the proposition. It is just that the considerations of the Euler characteristic used in the proof do not suffice to rule out these cases. In fact these cases will be ruled out by direct examination in $\S 9$.

ProOF OF (8.3). The formula $(*) \chi(X)=\sum\left(\chi\left(F_{i}\right)+\delta\left(F_{i}\right)\right)$, where $\chi(\cdot)$ signifies the topological Euler characteristic, $\delta\left(F_{i}\right)$ is Serre's higher ramification invariant for the fiber $F_{i}$, and the sum is over the fibers of $X$, is well known (cf. [Do]). But $X$ is rational so $\chi(X)=12$. Also, for any fiber $F_{i}, \chi\left(F_{i}\right)=\mu_{i}+\varepsilon_{i}$, where $\mu_{i}$ is the number of components of $F_{i}$ (not counting multiplicity) and $\varepsilon_{i}$ is either $-1,0$, or 1 , according to whether $F_{i}$ is smooth, of multiplicative type, or of additive type. So by substitution into $(*)$ we get

$$
12=\sum\left(\left(\mu_{i}+\varepsilon_{i}\right)+\delta\left(F_{i}\right)\right) \geq c+1+n+\alpha+\sum \delta\left(F_{i}\right),
$$

where $n$ is the number of $(-2)$-curves on $X, \alpha$ is the number of reducible fibers of additive type other than $F$, and $c=0$ if $F$ is reducible and $c=1$ if $F$ is irreducible. But by (7.4) we have $\eta(X)+r-1=n$ and by (7.3) and (8.1) we have $\eta(X)=\eta\left(X_{L}\right)=9$, so $(* *)$ becomes

$$
3 \geq c+r+\alpha+\sum \delta\left(F_{i}\right)
$$

Since $c+\alpha \geq 1$, we see that $r \leq 2$, and since $\eta(X)=9$ we see that $r \geq 1$.

Now $\eta(X)=9$ and $r=1$ implies that the unique reducible fiber $G$ has type either $A_{8}, E_{8}$, or $D_{8}$. By (7.3) the corresponding reducible fiber $G_{L}$ of $X_{L}$ has the same intersection graph. By $(8.1), G_{L}$ is of additive type, thus ruling out type $A_{8}$. A check of Néron [N, p. 95] using Theorem 2 of $[\mathbf{O}]$ shows that $\delta(H)$ is positive for any fiber $H$ of $X$ of additive type and weight a power of $p$. Now if $F$ is irreducible, it must be of type II, and if $G$ is either $E_{8}$ or $D_{8}$, we get $13 \leq \sum\left(\left(\mu_{i}+\varepsilon_{i}\right)+\delta\left(F_{i}\right)\right)$, which violates $(* *)$. Thus $F$ must be reducible and since $r=1$, this means that $F$ and $G$ are the same fiber. By (8.1) $F$ has weight a power of $p$, so we have the following cases: $F$ has type $E_{8}$ and $p$ is 2 or 3 , or $F$ has type $D_{8}$ and $p$ is 2 .

Now consider the case $r=2$. Suppose that neither reducible fiber has additive type. The reducible fibers on $X_{L}$ corresponding via (7.3) to those on $X$ are of additive type with unchanged intersection graph and weight a power of $p$. This is only possible for a reducible fiber on $X$ not of additive type if it has type $A_{1}$ when $p=2$ or $A_{2}$ when $p=3$. But two fibers of these types do not give $\eta(X)=9$, so at least one of the reducible fibers has additive type. Since $F$ irreducible implies $c+r+\alpha \geq 4$ contradicting $(\dagger), F$ itself is a reducible fiber of additive type. Let the other reducible fiber be called $G$. If $G$ has additive type, then $c+r+\alpha=3$, so by $(\dagger)$ we see $\delta(F)+\delta(G)=0$. By our observation above, this is impossible. Thus $G$ is not of additive type, so $G$ is either of type $A_{1}$ if $p=2$ or $A_{2}$ if $p=3$. Using $\eta(X)=9$ and (8.1)(iii), we see that $F$ is, respectively, of type $E_{7}$ or $E_{6}$.

9. The proof of Theorem (0.1). Our proof of Theorem (0.1) depends on the following lemma. 


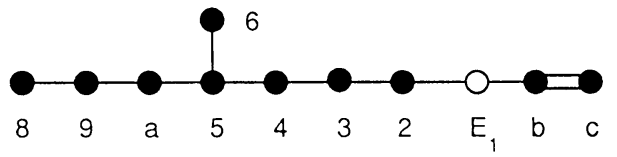

FIGURE 2

LEMMA (9.1). Let $X$ be a rational minimal elliptic surface with section, let $F$ be a fiber (necessarily of multiplicity 1), and suppose that there is a Halphen transform of $X$ for which $m F$ becomes a fiber of multiplicity $m$. Then there is always a choice of irreducible exceptional curve $E$ and an element $L \in \operatorname{Pic}^{0} F$ of order $m$ such that the Halphen transform $X_{L}$ associated to $(E, L)$ is elliptic.

Before giving the proof of (9.1), which will be completely constructive, we show how to derive $(0.1)$ from it.

PROOF OF (0.1). Suppose that there is a rational minimal elliptic surface having a fiber $m F$ of type $T$ and multiplicity $m$. Then $T$ can have at most 9 components by (5.1), and $m$ is the order in Pic $F$ of the normal bundle of $F$. If $F$ has additive type, then all nontrivial torsion has order equal to the characteristic. If $F$ has multiplicative type, then all torsion is relatively prime to the characteristic. If $F$ is neither of additive nor of multiplicative type, then $F$ is smooth. This proves the forward implication of $(0.1)$.

Conversely, suppose $T$ has no more than 9 components. Then there is by (6.1) a rational minimal elliptic surface $X$ with section on which there occurs a fiber $F$ of type $T$, and if $T$ is the type of a smooth fiber, we may arrange for $F$ not to be supersingular. Then given one of the three conditions (a), (b), or (c) of $(0.1)$, there is an element of Pic $F$ of order $m$. We wish to produce an elliptic surface having a fiber of type $m T$. If $m=1, X$ and $F$ give the desired example. So say $m$ is greater than 1. By (7.2), there is a Halphen transform of $X$ having a fiber of type $m T$. By (9.1), we may take this transform to be elliptic, thus giving the desired example and concluding the proof.

ProOF OF (9.1). Choose an irreducible exceptional curve $E$ on $X$. Then by (7.1) the Halphen transform $X_{L}$ of $X$ associated to $(E, L)$ is a numerically elliptic surface and $m F$ is a multiple fiber of multiplicity $m$, for any class $L$ of order $m$. If the characteristic, $p$, is neither 2 nor 3 , or if $F$ is not of additive type, then $X_{L}$ is in fact elliptic by $\S 1$ and (8.1), so we are done. So say $p$ is 2 or 3 , and $F$ is of additive type. If the Halphen transform of $X$ associated to $(E, L)$ were quasi-elliptic for a general element $L$ of $\operatorname{Pic}^{0} F$, then $X$ would be one of the surfaces enumerated in (8.3). We consider each of the five cases in turn.

Consider the case that $X$ has two reducible fibers, a fiber $F$ of type $E_{7}$ and a fiber $G$ of type $A_{1}$. By $(8.1)$ (iv) we see that $X$ has two irreducible exceptional curves. Call them $E_{1}$ and $E_{2}$. Since both are sections of the fibration, each meets the fibers $F$ and $G$ once each. Thus the intersection graph of the components of the curves $F, G$, and $E_{1}$ must be as in Figure 2, where the open dot represents $E_{1}$, and two edges connect the dots representing the components of $G$ to indicate that these curves meet twice. The symbols attached to the dots are merely for identification.

If $E$ is an irreducible exceptional curve and $D$ is a (-2)-curve with $E \cdot D=1$, then under the contraction of $E$ the image of $D$ becomes an irreducible exceptional curve 


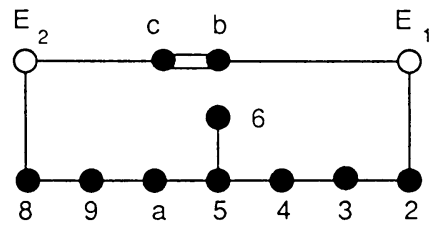

FIGURE 3

and may itself be contracted to a smooth point. Thus we can contract in order $E_{1}$ and the curves represented in Figure 2 by the dots $2,3,4,5, a$, and 9 , and by (4.2) there is still a pair of curves whose contraction results in a morphism to $\mathbb{P}^{2}$. However the only possible remaining exceptional curves are (the images of) $E_{2}$ and the curve denoted by dot 8 . Since $E_{2}$ originally gave a section, it must have originally met either the curve denoted by dot 8 or dot 2 . If we now contract (the curve denoted by) dot 8 , then $E_{2}$ cannot possibly still be an exceptional curve, and thus there are no exceptional curves, and the surface obtained under the sequence of contractions is not $\mathrm{P}^{2}$. If $E_{2}$ originally met dot 2 , then the image of $E_{2}$ under the sequence of contractions $E_{1}, 2,3,4,5, a, 9$ is again not still an exceptional curve. Thus $E_{2}$ must originally have met $F$ at $\operatorname{dot} 8$, whence after the sequence of contractions $E_{1}, 2,3,4,5, a, 9$ the image of $E_{2}$ can be contracted. Since there must remain an exceptional curve, the contraction of which gives $\mathbb{P}^{2}$, it is evidently the image of dot c. Therefore we see that by including $E_{2}$ in Figure 2 we must obtain Figure 3, which gives the intersection graph of all of the exceptional curves and (-2)-curves of $X$. It is now easy to verify that by contracting in order the curves $E_{1}, 2,3,4,5,6, E_{2}, 8$, and 9 , we obtain a morphism of $X$ to $\mathbb{P}^{2}$, and the image of $G$ is a cubic curve comprising an irreducible conic (the image of dot $b$ ) meeting a line (the image of $\operatorname{dot} c$ ) at two distinct points, while the image of $F$ is a line, taken with multiplicity 3 , tangent to the conic and meeting the linear component of the image of $G$ away from the conic.

Thus $X$ comes from blowing up the base points of this plane pencil of cubics, and it is easy to check that projective coordinates can always be chosen so that this pencil is $\left\{x^{3}, z\left(x y+x z+y^{2}\right)\right\}$, where $x^{3}$ is the image in $\mathbb{P}^{2}$ of $F$ and $z\left(x y+x z+y^{2}\right)$ is the image of $G$. With these same coordinates,

$$
\left\{x^{6},\left(x y+x z+y^{2}\right)\left(z^{2}\left(x y+x z+y^{2}\right)+x^{3}\left(\alpha^{2} x+\alpha z\right)\right)\right\}
$$

is, for any nonzero value of the coefficient $\alpha$, the plane sextic pencil corresponding to a Halphen transformation of $X$ for which $x^{6}$ corresponds to the fiber $2 F$ and $\left(x y+x z+y^{2}\right)\left(z^{2}\left(x y+x z+y^{2}\right)+x^{3}\left(\alpha^{2} x+\alpha z\right)\right)$ corresponds to a fiber of type $A_{1}$, and hence the fibration is elliptic.

To check that this sextic pencil is indeed the plane pencil corresponding to a Halphen transform $X^{\prime}$ of $X$ one merely needs to check that the first eight base points of both the cubic and sextic pencils are the same (and in fact the blowings-up of these eight base points give the curves $\left.8,9, E_{1}, 2,3,4,5,6\right)$ and that both pencils have distinct ninth base points infinitely near the common eighth base point. It follows that $X^{\prime}$ is the Halphen transform obtained from $X$ by contracting $E_{2}$ and then blowing up a point $q$, where $q$ is not in the image of $E_{2}$ under the contraction but $q$ is on the curve denoted by dot 8 in Figure 3 . Since $q$ is not in the image of 


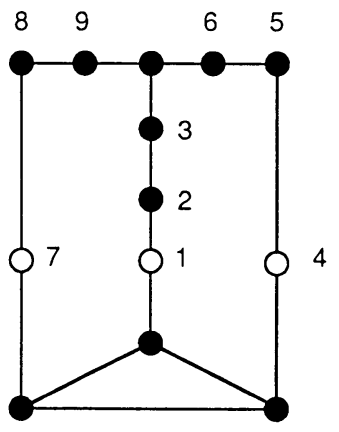

FIGURE 4

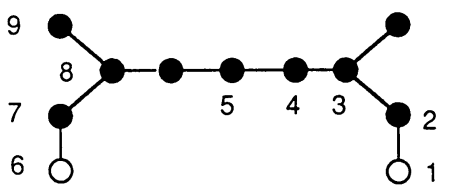

FIGURE 5

any irreducible exceptional curve of $X$, it follows from the proof of (7.3) that the fiber of $X^{\prime}$ corresponding to the element

$$
\left(x y+x z+y^{2}\right)\left(z^{2}\left(x y+x z+y^{2}\right)+x^{3}\left(\alpha^{2} x+\alpha z\right)\right)
$$

of the sextic pencil is either $A_{1}$ or $A_{1}^{*}$. Since the components $\left(x y+x z+y^{2}\right)$ and $\left(z^{2}\left(x y+x z+y^{2}\right)+x^{3}\left(\alpha^{2} x+\alpha z\right)\right)$ meet at the two distinct points with coordinates $x=1, z=\alpha$, and $y$ a solution of $y^{2}+y+\alpha=0$, and these points are away from the base points of the pencil we see that the fiber must be $A_{1}$.

We now consider, in less detail, the case that $X$ has a fiber $F$ of type $E_{6}$ and a fiber $G$ of type $A_{2}$. As above, we see the fibration on $X$ has three irreducible exceptional curves. An argument similar to that used above shows that the intersection graph of exceptional curves and (-2)-curves on $X$ must be as in Figure 4, the dots numbered in the order of contraction. It is easy to verify that the plane pencil of cubics which is the image of the elliptic fibration under the morphism to $\mathbf{P}^{2}$ given by these contractions can always be written in projective coordinates as $\left\{z^{3}, x y(x+z+y)\right\}$, where $z^{3}$ is the image in $\mathrm{P}^{2}$ of $F$ and $x y(x+z+y)$ is the image of $G$. With these same coordinates,

$$
\left\{z^{9}, x y\left(x^{2} y^{2}(x+z+y)^{3}-\alpha^{2} z^{6}((1-\alpha) x+(1+\alpha) y+z)\right)\right\}
$$

is for any scalar $\alpha \neq 0$ a plane nonic pencil corresponding to an elliptic Halphen transformation of $X$ for which the fiber $3 F$ corresponds to $z^{9}$ and a fiber of type $A_{2}$ corresponds to $x y\left(x^{2} y^{2}(x+z+y)^{3}-\alpha^{2} z^{6}((1-\alpha) x+(1+\alpha) y+z)\right)$.

Consider now the case that $X$ has a fiber $F$ of type $D_{8}$. Then the intersection diagram of the exceptional and (-2)-curves is as in Figure 5, and the plane pencil corresponding to the fibration on $X$ can always be put into the form $\left\{x z^{2}, x y^{2}+\right.$ $\left.x^{2} z+x y z+\alpha z^{3}\right\}$, where $\alpha$ is some nonzero scalar. A sextic pencil corresponding to an elliptic Halphen transform is $\left\{x^{2} z^{4}, x^{3} z^{3}+\gamma\left(x y^{2}+x^{2} z+x y z+\alpha z^{3}\right)^{2}+\delta\left(x^{2} y^{4}+x^{4} z^{2}+x^{2} y z^{3}+\alpha^{2} z^{6}+\alpha x z^{5}\right)\right\}$ 


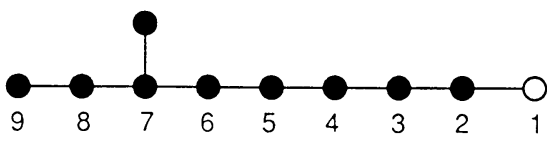

FiguRE 6

where $\gamma$ is $\left(\delta^{3}+1\right) /\left(\delta^{2}+1\right)$, and $\delta$ is any nonzero scalar other than 0 or 1 .

Lastly, we need to consider the case that $X$ has a fiber of type $E_{8}$. In this case there is only one irreducible exceptional curve, call it $E$, and the intersection graph of it and the $(-2)$-curves is as in Figure 6, and contracting the curves in the order indicated in the figure gives a morphism to $\mathbb{P}^{2}$. It follows that $X_{L}$ has the same intersection graph, and thus the corresponding sequence of contractions gives a morphism of $X_{L}$ to $\mathbb{P}^{2}$. Thus the image of $p F$ under this morphism is a line, which we may consider to be the line at infinity, taken with multiplicity $3 p$. The image of a general irreducible fiber is a curve of degree $3 p$ that is smooth except for a single singularity at infinity.

Suppose that $X_{L}$ is quasi-elliptic. Then the curve of cusps is the exceptional curve, and the image $C$ in $\mathbb{P}^{2}$ of an irreducible fiber is, apart from the cusp at infinity, isomorphic to the affine line $A^{1}$. Now from $[\mathbf{G a}]$ we see that if $z=0$ is the line at infinity, coordinates can be chosen so that the equation of $C$ is $\left(y^{3}-x^{2} z\right)^{2}-c y z^{5}$ if the characteristic 2 and $\left(y^{3}-x^{2} z\right)^{3}-c x z^{8}$ if the characteristic is 3 , for some nonzero scalar $c$. Thus the image of the fibration on $X_{L}$ is a plane pencil given by $C$ and $z^{3 p}$. The cubic plane pencil associated to the fibration on $X$ is, of course, the pencil of cubics whose first eight base points are the same as those of the pencil obtained from $X_{L}$. But a direct computation using the equations for the $3 p-i c$ pencil shows that the cubic pencil obtained is $\left\{z^{3}, y^{3}-x^{2} z\right\}$, which gives rise to a quasi-elliptic pencil, not an elliptic pencil as $X$ is supposed to have. Thus $X_{L}$ can never be quasi-elliptic if $X$ is elliptic.

ACKNOWLEDGEMENTS. We wish to thank M. Artin for suggesting the method of proof of (8.2) and D. Saltman for a number of helpful conversations and the referee for his careful reading of the paper.

\section{REFERENCES}

[B-M] E. Bombieri and D. Mumford, Enriques classification of surfaces in characteristic p. II, Complex Analysis and Algebriac Geometry, Cambridge, Univ. Press, 1977, pp. 23-42.

[Do] I. Dolgachev, The Euler characteristic of a family of algebraic varieties, Math. USSR Sb. 18 (1972), 303-319.

[DR] P. Deligne and M. Rapoport, Las schemas de modules de courbes elliptiques, Antwerp Summer School, Springer Lecture Notes in Math., vol. 349, Springer-Verlag, Berlin and New York, 1973, pp. 143-316.

[Ga] R. Ganong, Kodaira dimension of embeddings of the line in the plane, J. Math. Kyoto Univ. 25 no. 4 (1985), 649-657.

[Ha] R. Hartshorne, Algebraic geometry, Graduate Texts in Math., no. 52, Springer-Verlag, Berlin and New York, 1977, p. 496.

[Ka] T. Katsura, On Kummer surfaces in characteristic 2, Proc. Internat. Sympos. on Algebraic Geometry (Kyoto Univ., Kyoto, 1977), Kinokuniya Bookstore, Tokyo, 1978, pp. 525-542.

[Ko] K. Kodaira, On compact analytic surfaces. II, Ann. of Math. (2) 77 (1963), 563-626.

[KU] T. Katsura and K. Ueno, Multiple singular fibers of type $\mathbf{G}_{a}$ of elliptic surfaces in characteristic $p$, preprint, 1985 . 
[L1] W. E. Lang, Review of T. Katsura's paper On Kummer surfaces in characteristic 2 (see above citation). MR 82d: 14022.

[L2] _ Q Quasi-elliptic surfaces in characteristic 3, Ann. Sci. École Norm. Sup. (4) 12 (1979), 473-500.

[L3] $\ldots$, On the Euler number of algebraic surfaces in characteristic p, Amer. J. Math. 108 (1980), 511-516.

[L4] __ An analogue of the logarithmic transform in characteristic p, Proc. 1984 Vancouver Summer Conf. on Algebraic Geometry, Canad. Math. Soc. Conf. Proc., vol. 6, 1986, pp. 337-340.

[M] Yu. Manin, Cubic forms: algebra, geometry, arithmetic, North-Holland, Amsterdam, 1974.

[Mi] J. S. Milne, Étale cohomology, Princeton Univ. Press, Princeton, N.J., 1980, xiii and 323.

[Mu] D. Mumford, Enriques classification of surfaces in char $p$. I, Global Analysis, Papers in Honor of K. Kodaira, 1969, pp. 325-339.

[N] A. Néron, Modèles minimaux des variétés abéliennes sur les corps locaux et globaux, Inst. Hautes Études. Sci. Publ. Math. 21 (1964).

[O] A. P. Ogg, Elliptic curves and wild ramification, Amer. J. Math. 89 (1967), 1-21.

[R] M. Raynaud, Specialisation du functeur de Picard, Inst. Hautes Études Sci. Publ. Math. 38 (1970), 27-76.

[RS] A. N. Rudakov and I. R. Shafarevich, Supersingular K3 surfaces over fields of characteristic two, Math. USSR Izv. 13 (1979), 147-165.

Department of Mathematics and Statistics, UNiversity of Nebraska, LinCOLN, NEBRASKA 68588-0323

Department of Mathematics, University of Minnesota, Minneapolis, MinNESOTA 55455 